\title{
Simulation-aided investigation of beam hardening induced errors in CT dimensional metrology
}

\author{
Ye Tan ${ }^{1,2}$, Kim Kiekens $^{1,2}$, Frank Welkenhuyzen ${ }^{2}$, J. Angel $^{3}$, L. De Chiffre ${ }^{3}$, Jean-Pierre Kruth ${ }^{2}$ \\ and Wim Dewulf ${ }^{1,2, *}$ \\ ${ }^{1}$ KU Leuven, Campus Group T, Andreas Vesaliusstraat 13, 3000 Leuven, Belgium \\ ${ }^{2}$ KU Leuven, Mechanical Engineering Department, Celestijnenlaan 300B, 3001 Heverlee, Belgium \\ ${ }^{3}$ Technical University of Denmark, Department of Mechanical Engineering, Building 425, Produktionstorvet, 2800 Kgs. \\ Lyngby, Denmark \\ *Wim Dewulf / E-mail: Wim.Dewulf@kuleuven.be
}

\begin{abstract}
Industrial X-ray CT systems are increasingly used as dimensional measuring machines. However, micron level accuracy is not always achievable yet. The measurement accuracy is influenced by many factors, such as workpiece properties, $\mathrm{X}$-ray voltage, filter, beam hardening, scattering and calibration methods [1-4]. Since most of these factors are mutually correlated, it remains challenging to interpret measurement results and to identify the distinct error sources. Since simulations allow isolating the different affecting factors, they form a useful complement to experimental investigations.

Dewulf et.al [5] investigated the influence of beam hardening correction parameters on the diameter of a calibrated steel pin in different experimental set-ups. It was clearly shown that inappropriate beam hardening correction can result in significant dimensional errors. This paper confirms these results using simulations of a pin surrounded by a stepped cylinder: a clear discontinuity in the measured diameter of the inner pin is observed where it enters the surrounding material. The results are expanded with an investigation of the beam hardening effect on the measurement results for both inner and outer diameters of the surrounding stepped cylinder. Accuracy as well as the effect on the uncertainty determination are discussed. The results are compared with simulations using monochromatic beams in order to have a benchmark which excludes beam-hardening effects and X-ray scattering. Furthermore, based on the above results, the authors propose a case-dependent calibration artefact for beam hardening correction and edge offset determination.

In the final part of the paper, the investigations are expanded with experiments of a new set-up that includes non-cylindrical features; the effectiveness of the proposed calibration artefact is also studied.
\end{abstract}

Keywords: simulation, beam hardening, X-ray Computed Tomography, dimensional metrology

\section{INTRODUCTION}

1.1 Beam hardening effect

Most industrial X-ray tubes generate a polychromatic X-ray beam which is characterized by a continuous energy spectrum with certain bandwidth. Due to the energy dependent attenuation, lower energy (soft) X-rays are more easily and rapidly absorbed than high energy (hard) X-rays as the beam passes through a workpiece. Thus, the average frequency of the X-rays is shifted in the direction of higher energy during the propagation process; this is referred to as "hardening" of the X-ray beam. Because of the beam hardening $(\mathrm{BH})$ effect, the X-ray attenuation is not strictly linearly related to the penetrated material thickness [5] (Fig. 1).

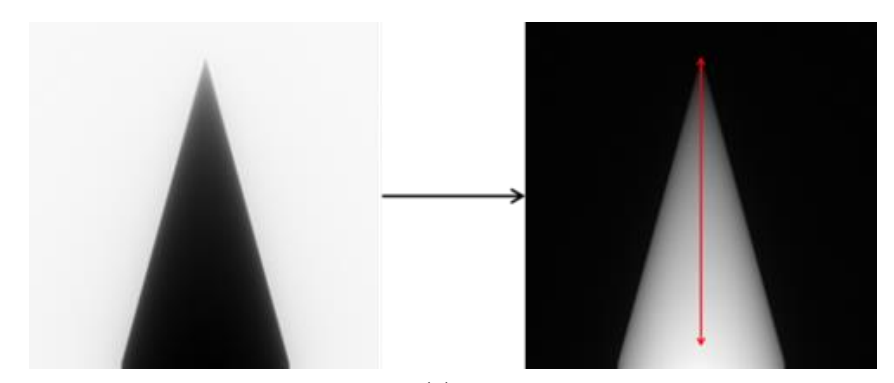

(a)

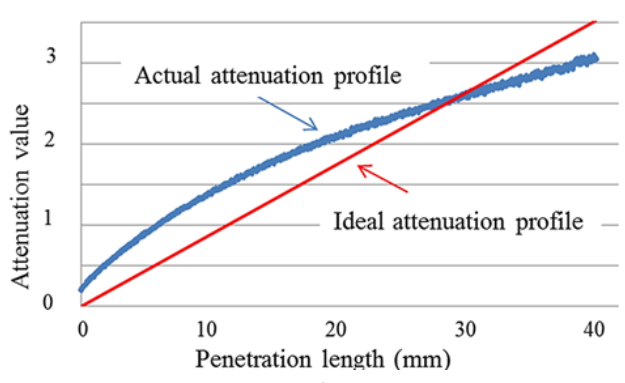

(b)

Fig. 1 (a) 2D X-ray projection image of a steel cone: pixel intensity values (gray values) are converted to X-ray attenuation values (the ability of attenuating X-ray beams); (b) Attenuation profile along the "red line" in (a). 


\subsection{Beam hardening correction}

Most reconstruction algorithms presume a linear relationship between the X-ray attenuation and the X-ray penetration length; as a result, non-linear artifacts such as cupping effects (Fig. 2) and dark streaks (Fig. 3) are generated after reconstruction due to the $\mathrm{BH}$ effect. These artifacts strongly degrade image quality, and hinder accurate material analysis and defect detection.
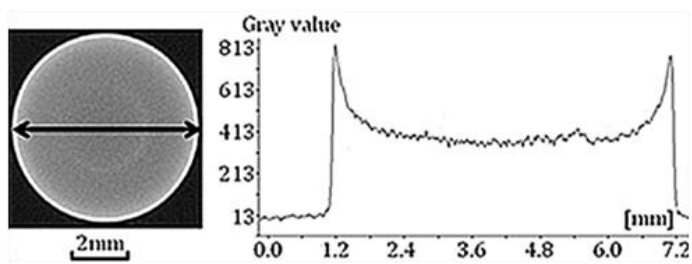

Fig. 2 (left) reconstructed slice of a steel cylinder and corresponding grey value profile along the arrow line [5].

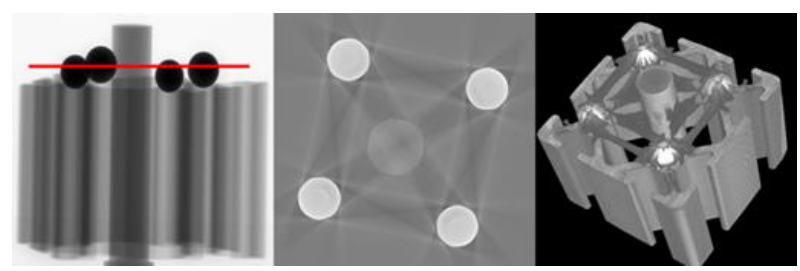

Fig. 3 (right) (a) 2D X-ray image of an aluminum profile with four steel spheres; (b) streak artifacts visible in a reconstructed CT slice of the red section in (a); (c) 3D CT voxel model of the objects [5].

Many approaches have been developed for beam hardening correction (BHC); ranging from the most basic hardware filtration method to advanced iterative reconstruction algorithms [6,7]. However, taking the computational cost and effectiveness into consideration, the linearization technique using pre-defined polynomials combined with hardware prefiltration is favored by most industrial users. Currently, polynomials up to the fourth order are being used:

$$
\mathrm{Y}=\mathrm{a}\left(\mathrm{b}+\mathrm{cX}+\mathrm{dX}^{2}+\mathrm{eX}^{3}+\mathrm{fX}^{4}\right)
$$

The polynomial is the relation between the ideal attenuation values without beam hardening $\mathrm{Y}$ and the measured attenuation values X; and "a" through " $\mathrm{f}$ " represent coefficients that can be fine-tuned depending on the severity of BH effect. A number of experience based BHC presets (up to second order) are listed in Table 1 [5].

\begin{tabular}{ccccccc}
\hline Presets & \multicolumn{6}{c}{ Parameters } \\
\cline { 2 - 6 } & $\mathrm{a}$ & $\mathrm{b}$ & $\mathrm{c}$ & $\mathrm{d}$ & $\mathrm{e}$ & $\mathrm{f}$ \\
\hline 1 & 1 & 0 & 1 & 0 & 0 & 0 \\
2 & 1 & 0 & 0.75 & 0.25 & 0 & 0 \\
3 & 1 & 0 & 0.5 & 0.5 & 0 & 0 \\
\hline
\end{tabular}

Table 1. Experience based BHC presets. Nr.1 keeps the original data; Nr.2 applies moderate BHC; Nr. 3 applies the severest BHC.

BHC can largely improve the image quality and makes the grey value of the same material appear uniform after reconstruction. On the other hand, the BH effect enhances edge contrast [8], since the material outer surface experiences a rapid gray value change; which is beneficial for material outer surface determination. The question remains whether BHC is beneficial for dimensional metrology applications.

\subsection{Initial experimental investigation: the influence of $\mathrm{BHC}$ on internal dimensions}

The main power of industrial CT for dimensional metrology relies on its ability to measure internal features non-destructively. Thus, a simple setup (Fig. 4) has been used to investigate the influences of BHC on the measurement accuracy and uncertainty of internal dimensions. The main machine settings for scanning this setup and data analysis software are listed in Table 2 .

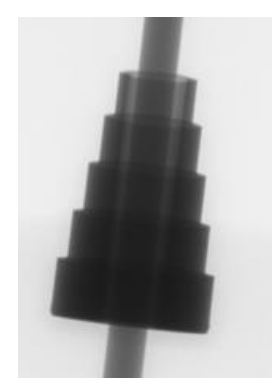

Fig. 4 2D X-ray projection image of the test setup: a calibrated stainless steel pin ( surrounded by a stainless steel step cylinder. Thus, the middle part of the inner pin can be considered as an "internal" feature. 


\begin{tabular}{cccccc}
\hline Voltage $(\mathrm{KV})$ & Voxel Size $(\mu \mathrm{m})$ & Current $(\mu \mathrm{A})$ & $\begin{array}{c}\text { Copper filter } \\
(\mathrm{mm})\end{array}$ & Reconstruction & Segmentation \\
\hline 200 & 42 & 180 & 2 & FBP by CTPro XT & $\begin{array}{c}\text { Local adaptive thresholding by } \\
\text { VGStudio MAX 2.2 }\end{array}$ \\
\hline
\end{tabular}

Table 2. Main machine settings and data analysis software for the setup in Fig. 4.

The original scan data (2D X-ray projections) are initially reconstructed using BHC preset Nr.1 (no correction) and Nr.2 (moderate correction). After local adaptive surface determination, proper object alignment and voxel size correction, the diameter of the middle pin is measured at equidistant slices from top to bottom. Fig. 5 plots the dimensional error (difference between the CT measured value and the reference value of $\varnothing 4 \mathrm{~mm}$ ) against the slice number (position where the measurements are taken: from top to bottom).

The measurements confirm the results reported by Dewulf et al. [5]: local dimensional variations are observed when the pin enters or leaves the surrounding step cylinder. Such discontinuity is around $4 \mu \mathrm{m}$ if no BHC is applied, and around $10 \mu \mathrm{m}$ when applying BHC preset Nr.2 (as listed in Table 1). The major cause is that the surrounding material acts as an additional filter. As a result, the level of $\mathrm{BH}$ effect along the middle pin differs depending on the location. The difference of $\mathrm{BH}$ level results in a difference of edge offsets; this appears as a discontinuity seen in Fig. 5. Non-proper BHC and especially overcorrection can enlarge the difference between the inner and outer edge offsets and worsen the measurement uncertainty.

It can be noticed that the direction of the discontinuity is inverted for BHC preset Nr.1 and Nr.2. It indicates that BHC preset Nr.1 yields insufficient correction, whereas BHC preset Nr.2 yields over-correction; this suggests that it is possible to eliminate these discontinuities by fine tuning the coefficients of the BHC polynomial. After 2 iterations, optimal BHC coefficients are obtained: $a=1, b=e=f=0, c=0.85$ and $d=0.15$ (see Formula 1). Significant improvement of the dimensional measurement results is achieved. Apart from an edge offset (which can be corrected by applying a correction term, the overall dimensional errors are within $2.5 \mu \mathrm{m}$ (see purple line in Fig.5). This is very close to the dimensional tolerance of the pin: $\pm 1 \mu \mathrm{m}$.

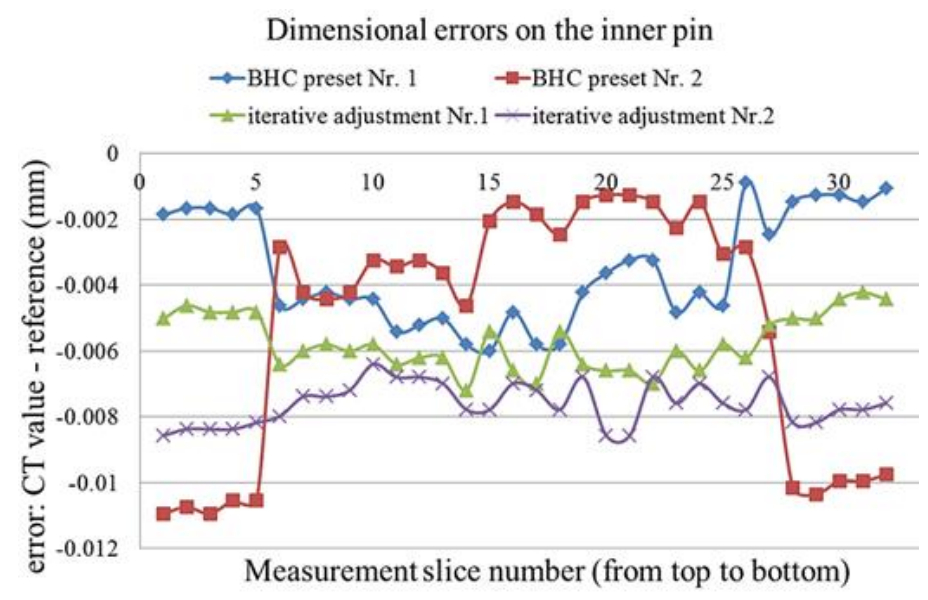

Fig. 5 Comparison of dimensional measurement errors on the inner pin (shown in Fig. 4) using different BHC coefficient sets. Attention: BHC preset Nr.1 - no BH correction.

\subsection{Note on the influence of X-ray scattering}

Although photoelectric absorption is the dominant process for $\mathrm{x}$-ray energies up to about $500 \mathrm{keV}$, Compton scattering still plays an important role in X-ray attenuation and is one of the major error sources for CT dimensional metrology applications using cone beam X-ray and flat panel detector. It occurs when the incident X-ray photon is deflected from its original path by an interaction with an electron. The electron gains energy and is ejected from its orbital position. The X-ray photon loses energy due to the interaction but continues to travel through the material along an altered path [9]. Incident X-ray photons reaching the detector will result in an overestimation of the registered total intensity in each projection which is equivalent to an underestimation of attenuation; this can also lead to image artifacts such as "cupping" in homogeneous objects or dark streaks between image regions of high attenuation [10]. Thus, the image artifacts of beam hardening and X-ray scattering are often mixed; and beam hardening correction also partly corrects for X-ray scattering. Since this hampers isolating and investigating the influence of the beam hardening effect on dimensional measurements, simulations are used in the next section. 


\section{SIMULATION-BASED INVESTIGATION OF THE INFLUENCE OF BEAM HARDENING}

In order to clearly identify the influence of the BH effect and of BHC on the dimensional measurement results, it is important to eliminate other influencing factors, such as X-ray scattering, machine axes misalignment and focal spot drifting. These conditions are almost impossible to achieve experimentally, but can be easily realized by X-ray CT simulation [11, 12]. The simulation software by Welkenhuyzen et al. [11] has been used throughout the reported investigations. Two CT scans of a steel testing object (shown in Fig. 6) are simulated: the first CT simulation is performed with a monochromatic spectrum, to serve as a benchmark; the second CT simulation uses polychromatic spectrum which will result in the beam hardening effect after FBP reconstruction. Both simulations eliminate X-ray scattering, such that the influence of the beam hardening effect on the accuracy and uncertainty of dimensional measurements can be studied independently. The applied simulation parameters and analysis software are listed in Table 3.

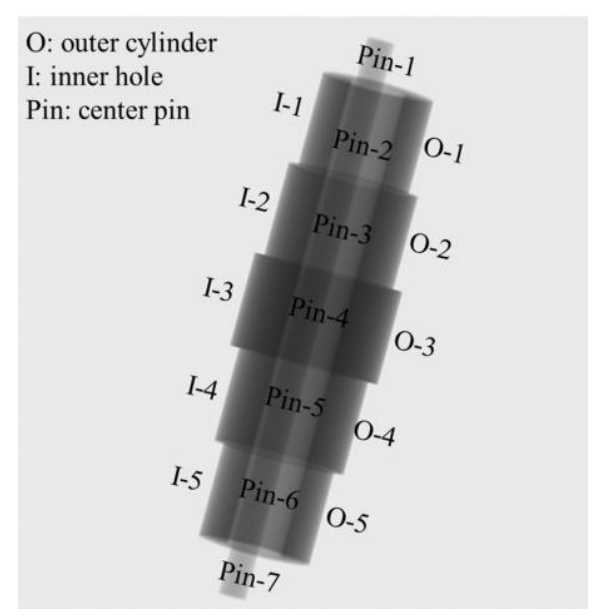

(a)

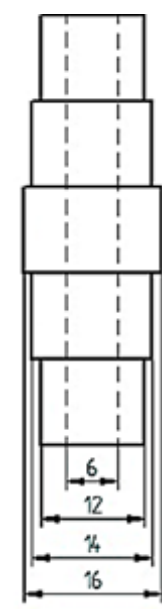

(b)

Fig. 6 (a) Simulated 2D projection image of the testing object: a symmetric steel hollow step cylinder and an inner steel pin (Ø3mm, the dimensional tolerance for the real pin is $\pm 1 \mu \mathrm{m}$ ); (b) Designed dimensions of the outer cylinder.

\begin{tabular}{ccccc}
\hline Voltage $(\mathrm{KV})$ & Voxel size $(\mu \mathrm{m})$ & Copper filter $(\mathrm{mm})$ & Reconstruction & Segmentation \\
\hline 210 & 39 & 2 & FBP by CTPro XT & Local adaptive thresholding by VGStudio \\
& & & 3.1 .2 & MAX 2.2 \\
\hline
\end{tabular}

Table 3. Main simulation parameters and data analysis software for the setup in Fig. 6

After local adaptive thresholding and setting up the work coordinates, the inner pin diameter is measured as circles at equidistance slices in the same way as described in Section 1.3. Moreover, both the inner pin and the hollow step cylinder (inner hole and outer step cylinder) are divided into several sections as indicated in Fig. 6(a), and their diameters are measured as cylinders. The simulated CT scan data (using polychromatic X-ray spectrum) are initially reconstructed using BHC preset Nr.1 (no correction) and Nr.2 as listed in Table 1. By minimizing the size of the discontinuity at the inner pin, optimal BHC coefficients are found after a few iterations. The dimensional measurement results are shown in Fig. 7 and 8.

In general, the simulated measurement results coincide with the real measurements. Local dimensional variations are observed when the pin enters and leaves the surrounding step cylinder when a polychromatic X-ray source is simulated, hence involving beam hardening. Figure 7 also reveals that, even under the ideal conditions (monochromatic source, no Xray scattering, no machine axis misalignment...), the commonly accepted thresholding technique (which is based on the local gradient variation) will still introduce an edge offset. Thus, it is necessary to experimentally calculate the case dependent edge offset for CT dimensional metrology applications. Furthermore, the previous conclusion on the effect of BHC is also verified by the simulation. When we don't correct or over-correct for the BH effect, an additional difference on edge offsets for inner and outer features will be introduced. Luckily, it is possible to eliminate these local dimensional variations by the optimizing the BHC coefficients.

Figure 8 shows the dimensional measurement results for the outer step cylinders, inner hole and pin measured at different sections (as indicated in Fig. 6(a)) as separate cylinders instead of as diameters of circles on individual slices, as reported in e.g. Fig. 7. The results clearly confirm the previous conclusions and reveal additional messages: 
1. Even under the ideal condition (monochromatic spectrum, no X-ray scattering and machine axis misalignment...), edge offset still exists when using local adaptive thresholding method.

2. The previously mentioned discontinuities on the inner pin are again observed when measured as cylinders. It is around

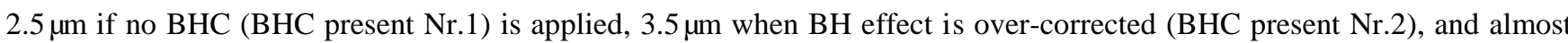
invisible when appropriate BHC (optimized BHC coefficients after 2 iterations) is applied or in case of a monochromatic source.

3. Theoretically, when there is an edge definition error, the edge offset on the outer step cylinders and the inner pin should follow the same trend, and the inner hole has the opposite value. However, beam hardening effect can strongly influence the direction and magnitude of the edge offsets. As shown in Fig. 8, if no BHC (BHC preset Nr.1) is applied, the edge offset for outer step cylinders are inverted; when the BH effect is over-corrected (BHC preset Nr.2), the direction of the outer step cylinder edge offset are corrected, but the error is much larger than before; when applying appropriate BHC, both the direction and the magnitude of the edge offset are corrected: in this case, $2 \mu \mathrm{m}$ for solid outer surfaces and $-2 \mu \mathrm{m}$ for inner surfaces.

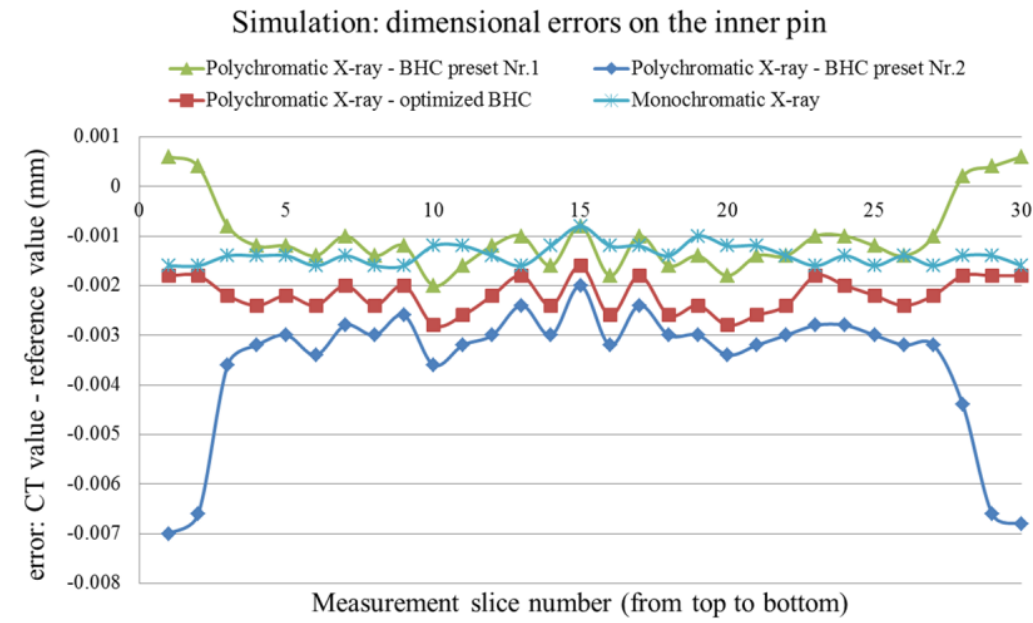

Fig. 7 Comparison of dimensional measurement errors on the inner pin (shown in Fig. 6) using different BHC coefficient sets. Attention: BHC preset Nr.1 means no correction.

Simulation: dimensional errors on the outer step cylinders, inner hole and pin measured at different sections as seperate cylinders

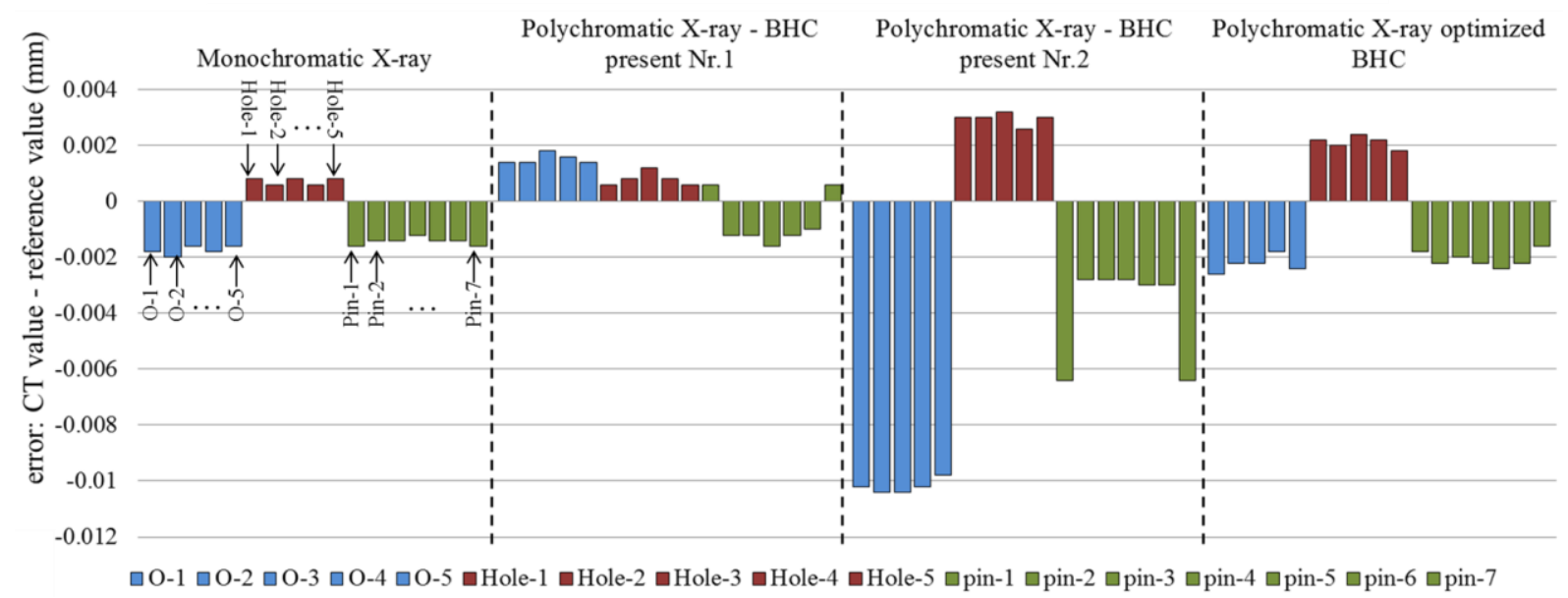

Fig. 8 Comparison of dimensional measurement errors on the outer step cylinder, inner hole and inner pin, measured at different sections as separate cylinders as indicated in Fig. 6(a). The coefficients for BHC preset Nr.1 and Nr.2 are listed in Table 1; the optimal BHC coefficients are: $\mathrm{a}=1, \mathrm{~b}=\mathrm{e}=\mathrm{f}=0, \mathrm{c}=0.9$ and $\mathrm{d}=0.1$ (see formula 1 and Table 1 for the exact meaning of "a" through " $\mathrm{f}$ "). Notice that BHC preset Nr.1 means no correction.

From the above results, we can conclude that appropriate BHC is useful for dimensional metrology applications. As shown in Fig. 7 and 8 , the non-systematic errors are reduced from $3 \mu \mathrm{m}$ to within $1 \mu \mathrm{m}$ by applying proper BHC; furthermore, after such BHC, it is much easier to compensate for the edge errors, because the edge offsets for inner and outer surfaces have similar magnitude and with opposite sign. However, one has to be very careful not to over-correct the BH effect. Otherwise, the local dimensional variations might increase. 


\section{EXPERIMENTAL VERIFICATION}

In addition to the simulation verification, the test object shown in Fig.6 was also manufactured, calibrated using a tactile $\mathrm{CMM}$, and measured on a $225 \mathrm{kV}$ industrial CT system. The machine settings for scanning this workpiece are listed in Table 4. The experiments go beyond the initial investigations reported in Section 1.3 by including also the dimensions of the external structure (stepped cylinder with through hole).

\begin{tabular}{cccccc}
\hline Voltage $(\mathrm{KV})$ & Voxel Size $(\mu \mathrm{m})$ & Current $(\mu \mathrm{A})$ & $\begin{array}{c}\text { Copper filter } \\
(\mathrm{mm})\end{array}$ & Reconstruction & Segmentation \\
\hline 210 & 39 & 195 & 2 & FBP by CTPro XT & $\begin{array}{c}\text { Local adaptive thresholding by } \\
\text { VGStudio MAX 2.2 }\end{array}$ \\
\hline
\end{tabular}

Table 4. Main machine settings and data analysis software for the setup in Fig. 6.

The diameter of the center pin is measured in the same way as described in Section 1.3 and 2 (measured as circles at equidistant slices from top to bottom). The CT scan data are initially reconstructed using BHC preset Nr.1 (no correction) and Nr.2; the local discontinuities at the inner pin are again observed and used as a tool to define the optimal coefficients for the BHC polynomial: by iteratively adjusting the coefficients to minimize these local variations. In this case, the optimized coefficients are: $a=1, b=e=f=0, c=0.82$ and $d=0.18$. As shown in Fig. 9, the local dimensional variations are reduced from $10 \mu \mathrm{m}$ (BHC preset Nr.1) to $4 \mu \mathrm{m}$. This is larger than the $1 \mu \mathrm{m}$ deviation which we have achieved with the simulation test (Fig.7), this might be due to the real CT scan also involve many other influencing factors, such as: X-ray scattering, focal spot drifting, machine axis misalignment, workpiece inaccuracy etc.

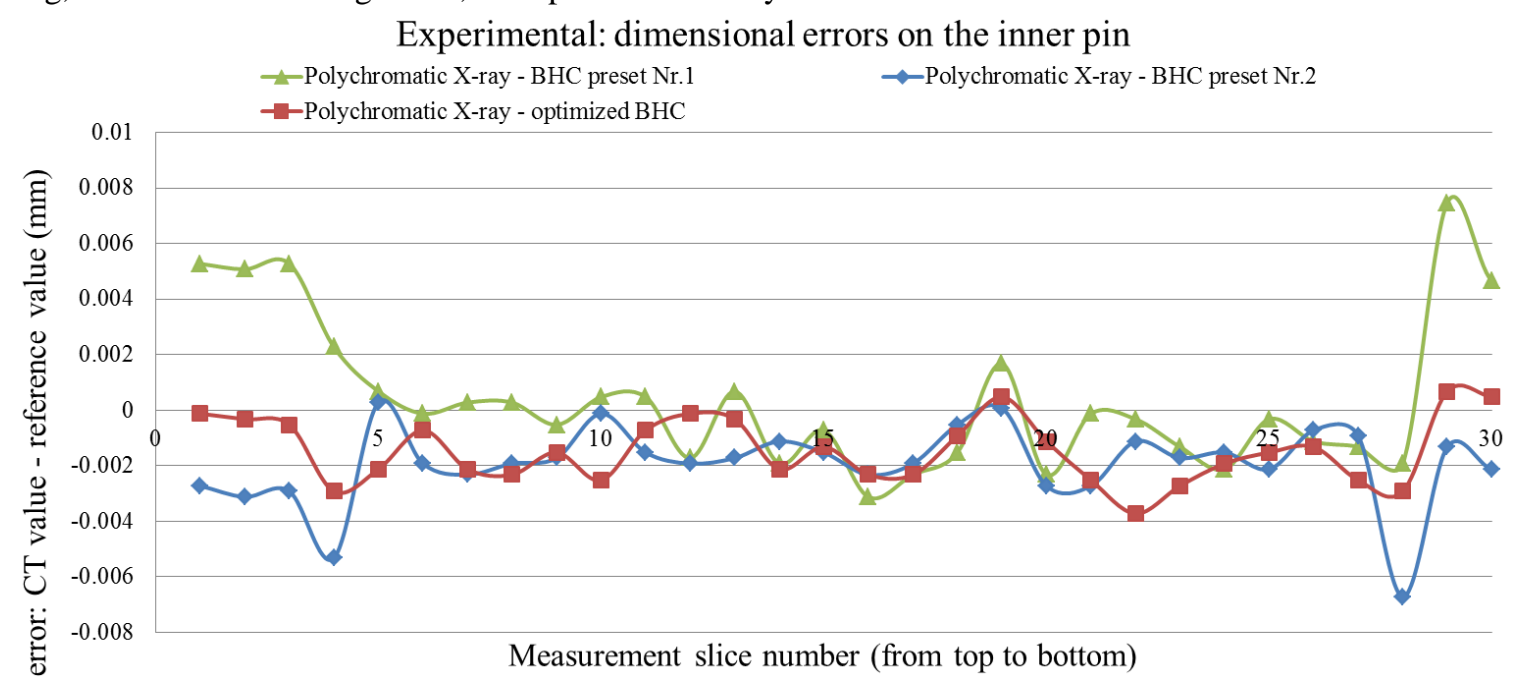

Fig. 9 Dimensional errors on the inner pin: its diameter is measured as circles from top to bottom at equidistance slices. The scan data are processed in three ways: without BHC (BHC preset Nr.1), with BH over correction (BHC preset Nr.2), and with fine-tuned BHC coefficients: $a=1, b=e=f=0, c=0.82$ and $d=0.18$.

As indicated in Fig.6 (a), this testing setup is further divided into different sections depending on the material penetration length: 5 sections on the outer step cylinders, 5 sections on the inner hole and 7 sections on the center pin. Their diameters are measured as cylinders and all measurement results are shown in Fig.10. Several conclusions can be drawn from this comparison:

1. Dimensional variations between different "sections" are observed when measured as cylinders. This non-systematical error reaches $8 \mu \mathrm{m}$ on the center pin when no BHC is applied (BHC preset Nr.1), and is reduced to $4 \mu \mathrm{m}$ when the scan data is processed with fine-tuned BHC coefficients. Moreover, the magnitude of the non-systematical errors on the outer step cylinders and inner hole are also reduced when applying appropriate BHC.

2. The material border will "shrink" (the outer diameters become smaller and inner hole become larger) depending on the level of BHC. In other words, BHC not only influences the non-systematic error, but also affects the direction and magnitude of the systematic edge offsets. 
3. Unlike plane distances, the edge dependent dimensions don't necessarily have the same edge offset. In this case, the edge offset for the inner hole is different from the one for the outer cylinders and center pin. However, applying appropriate BHC can reduce this difference.

4. These measurement results verify that the "optimized" BHC coefficients not only reduce the local dimensional variations on the inner pin, but also reduce other non-systematic errors and improve the overall measurement accuracy.

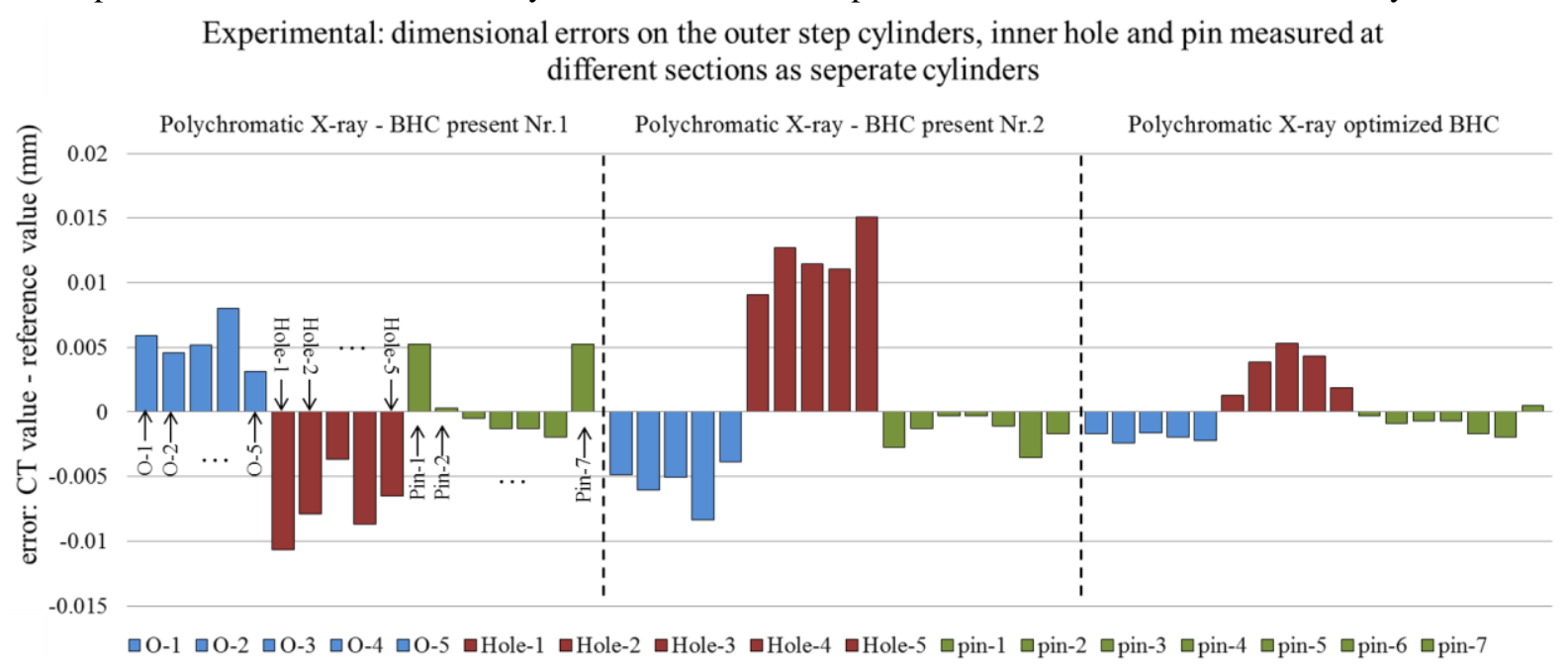

Fig. 10 Dimensional errors on the outer step cylinders, inner hole and center pin at different sections. The scan data are processed in three ways: without BHC (BHC preset Nr.1), with BH over correction (BHC preset Nr.2), and with fine-tuned $\mathrm{BHC}$ coefficients: $\mathrm{a}=1, \mathrm{~b}=\mathrm{e}=\mathrm{f}=0$, $\mathrm{c}=0.82$ and $\mathrm{d}=0.18$.

\section{WORKPIECE FOR BHC CALIBRATION}

\subsection{Basic concept}

There are methods for determining the "best" BHC polynomial coefficients, for example by looking at the horizontal center slice on the X-ray detector, where the sum of total attenuation along the central slice should be equal for each scan angle. However, this method requires perfect alignment between X-ray source and detector, plus the maximum penetration length be placed at the center line (to ensure the optimization calculation covers the entire data range: from air to maximum attenuation), these constraints are not very practical; moreover, such method also has difficulties when dealing with purely cylindrical objects, because it makes use of the difference between each projection images which is negligible for cylindrical objects.

As concluded from the previous sections, it is possible to optimize the coefficients of the BHC polynomial by minimizing the non-systematic dimensional errors on the center pin. It is also proven through both X-ray CT simulations and experimental approaches that appropriate BHC can regulate the edge offsets for both outer and inner surfaces, therefore improving the overall accuracy and uncertainty of dimensional measurement results. If we assume these optimal coefficients stay more or less constant as long as the scanned object consists of the same material, of similar size, and is scanned using the same machine settings; then, the combination of a center pin partly surrounded by another hollow object can be used as a calibration setup for BHC. Furthermore, the dimensional errors detected on the outer features (outer step cylinders, center pin) and inner features (inner hole) can give a good indication on the direction and magnitude of the edge error.

\subsection{Application verification}

\subsubsection{Workpiece description}

A steel step gauge developed at DTU (Fig. 11) is used to test the above mentioned functions of the calibration workpiece (shown in Fig. 6). Two types of plane distances are included on this object: unidirectional and bidirectional dimensions. As indicated in Fig. 11(b), unidirectional dimensions are not influenced by the material surface determination, thus are edge independent; whereas bidirectional dimensions are sensitive to the material surface determination and are edge dependent. Fig. 11(c) demonstrates two types of edge dependent plane distances on the step gauge. Along with the changing material edge, one type of distances will shrink whereas the other type of distances expands. 


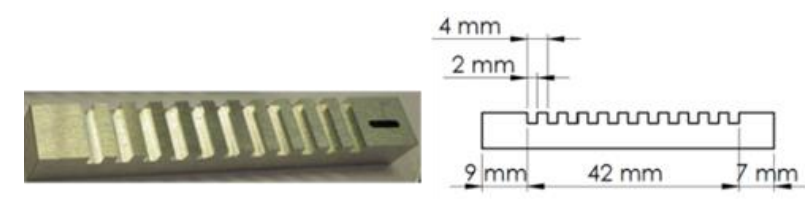

(a)

Groove 6

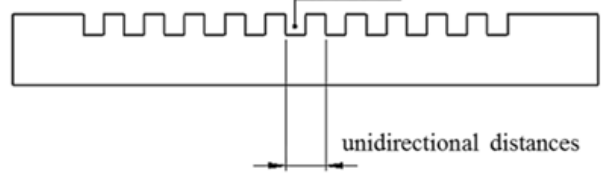

Groove 6

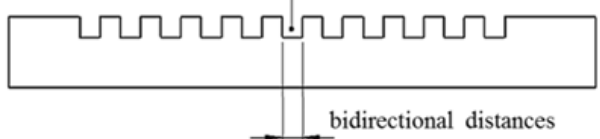

(b)

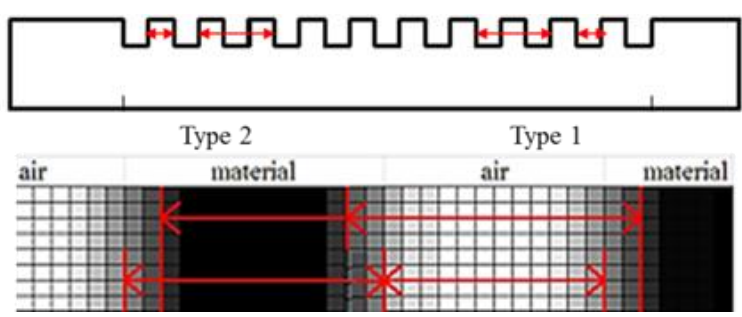

(c)

Fig. 11 (a) a photo of the step gauge and its designed dimensions; (b) indication of the unidirectional distance (edge independent) and bidirectional distance (edge dependent); (c) two types of edge dependent distances on the step gauge. Theoretically, type 1 and 2 have opposite edge offsets.

\subsubsection{Dimensional measurement results}

The step gauge is scanned using the same machine settings as the workpiece shown in Fig. 6, the scanning parameters and involved data analysis software are listed in Table 4. During reconstruction, the scan data are processed in two ways: without BHC (BHC preset Nr.1); and with "optimized" BHC polynomial coefficients (calculated in Section 3 - experimental verification using the measurement of object shown in Fig. 6). After local adaptive surface determination and voxel size correction, 10 unidirectional distances and 11 bidirectional distances are measured and compared with their reference values (tactile CMM measurement results), as shown in Fig. 12.

Several conclusions can be drawn from Fig. 12(a) - dimensional measurement results for unidirectional plane distances:

1. The non-systematic errors are slightly reduced (from $3 \mu \mathrm{m}$ range to $1.5 \mu \mathrm{m}$ range) when applying the "optimized" BHC polynomial coefficients $(a=1, b=e=f=0, c=0.82$ and $d=0.18$, same as for the workpiece in Fig. 6$)$.

2. There is still space for improvement; for example by reducing scattering noise, minimizing focal spot drifting and eliminating machine axes' misalignment etc.

The information that we get from Fig. 12(b) - dimensional measurement results for bidirectional plane distances are:

1. When measuring distances between parallel planes, the positive and negative edge offset errors often have similar absolute value. Thus, the sum of two distances, one with positive edge offset and the other one with negative edge offset, are often used for voxel size correction, because the edge errors will be eliminated. However, this method cannot be generalized for objects with other kinds of features. Taking the cylindrical object (Fig. 6) as an example, the edge offset for the inner hole doesn't necessarily equal to the outer cylinder's edge offset.

2. The optimized BHC polynomial coefficients calculated from a similar object (similar material, maximum penetration length and scanned using the same machine settings) can improve the dimensional measurement results; In this case, edge offset errors are reduced from $\pm 15 \mu \mathrm{m}$ to $\pm 7.5 \mu \mathrm{m}$.

3. Applying the edge offset terms calculated from the cylindrical object $(3.3 \mu \mathrm{m}$ and $-1.3 \mu \mathrm{m})$ can improve the dimensional measurement results but cannot completely eliminate the edge errors. Presumably this relates to the fact that both objects don't consist of twice exactly the same material (different types of steel). 


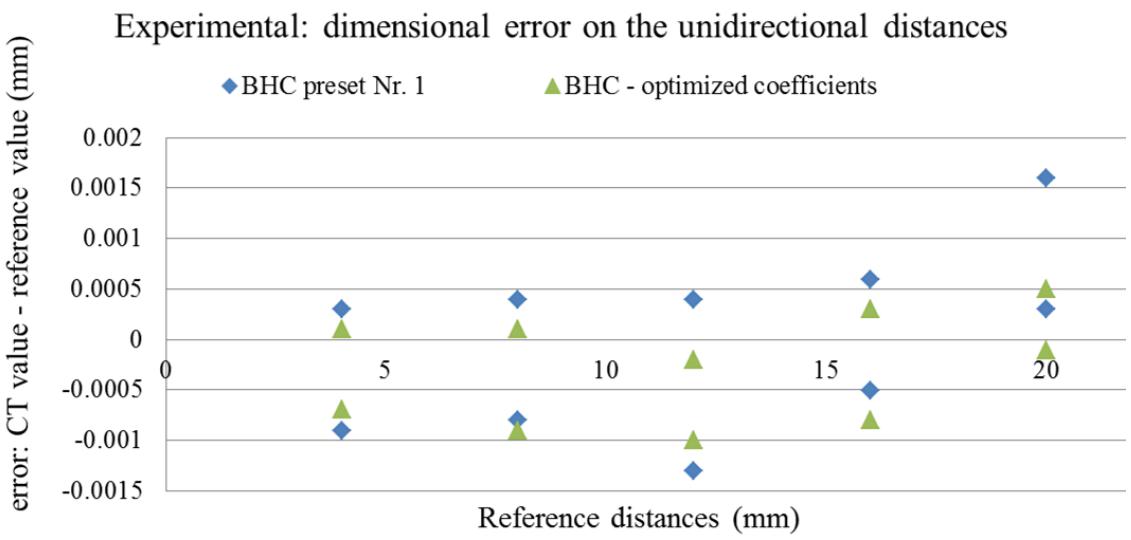

(a)

Experimental: dimensional error on the bidirectional distances

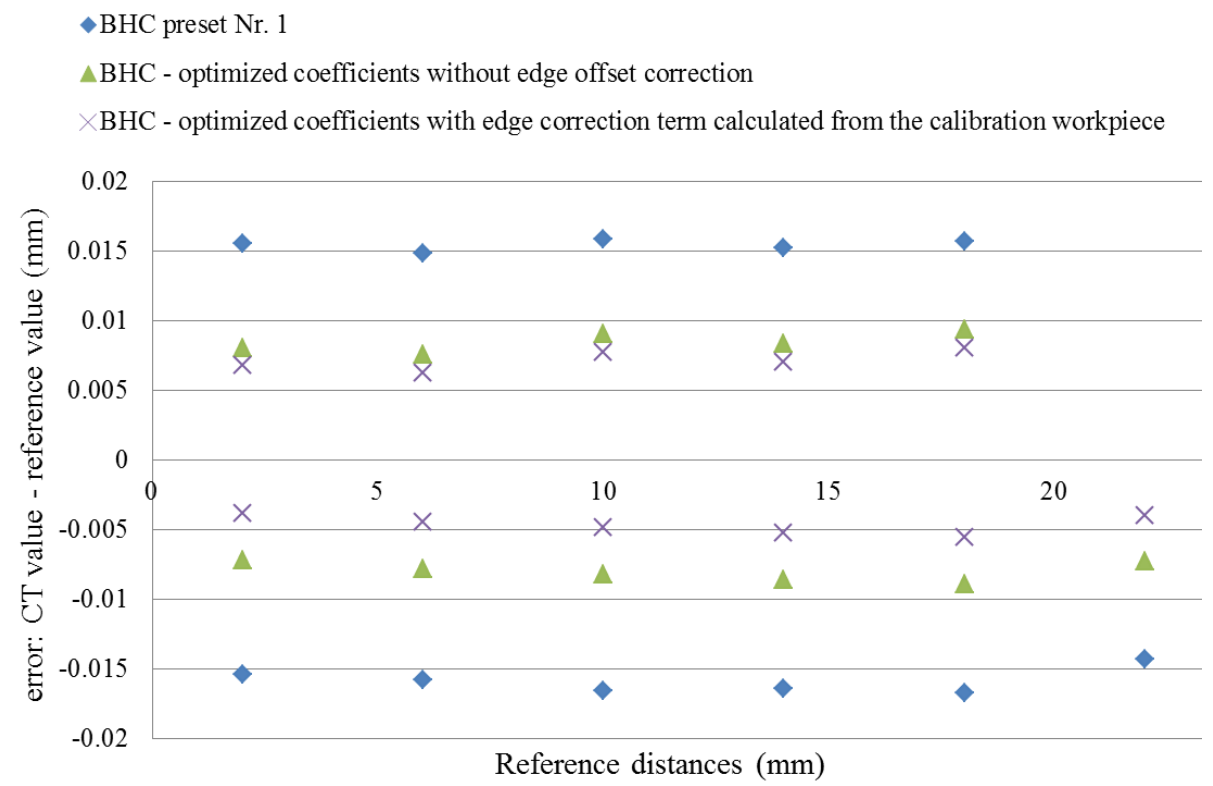

(b)

Fig. 12 (a) dimensional measurement results for 10 unidirectional distances: without BHC and with optimized BHC polynomial coefficients (b) dimensional measurement results for 11 bidirectional distances: without BHC, with optimized BHC polynomial coefficients and further improved by applying edge correction terms. The top half are "type 1" distances, the bottom half are "type 2" distances. Type 1 and 2 are as defined in Fig. 11(c).

\section{DISCUSSION AND CONCLUSION}

This paper investigated the influence of BH effect and BHC for CT dimensional metrology applications. The results verify our previous findings [5] which show that improper BHC can significantly worsen the accuracy and uncertainty of dimensional measurements. Both X-ray CT simulations and experiments verify that "optimal” BHC coefficients can be found by minimizing the local dimensional variations on the center pin. Moreover, it is also proven that applying appropriate BHC can improve the overall quality of dimensional measurements.

Furthermore, the setup shown in Fig. 6 is tested as a calibration object, for optimizing the coefficients of BHC polynomial and calculating the edge offset correction terms for a steel step gauge of similar size scanned under the same machine settings. On one hand, the optimized BHC coefficients turns out to be successful; on the other hand, the edge offset correction term calculated from the calibration object can reduce but not completely eliminate the edge errors for plane distances. This might be due to feature dependent errors, differences in X-ray scattering and other influencing factors.

It is found through our investigation that internal and external features often have different edge offsets, this might be due to BH effect, X-ray scattering and the applied surface determination algorithm. Thus, single edge correction term is insufficient for objects with complex internal and external features. 
It has to be mentioned that X-ray CT simulation plays an important role in helping us interpret our experimental results and identify the distinct error sources.

\section{ACKNOWLEDGEMENT}

The authors acknowledge the support of the Research Foundation Flanders (FWO) via project G.0711.11 N and G.0618.10 and of the Flemish Agency for Innovation by Science and Technology (IWT) via project TETRA 120167.

\section{REFERENCES}

1. Kruth JP, Bartscher M, Carmignato S, Schmitt R, De Chiffre L, Weckenmann A "Computed Tomography for Dimensional Metrology,” Annals of the CIRP, Manufacturing Technology Vol.60(2):821-842, 2011.

2. Bartscher M, Hilpert U, Goebbels J, Weidemann G "Enhancement and Proof of Accuracy of Industrial Computed Tomography (CT) Measurement,” Annals of the CIRP, Manufacturing Technology 56(1):495-498, 2007.

3. De Chiffre L., Hansen, H.N., Morace R.E., "Comparison of Coordinate Measuring Machines using an Optomechanical Hole Plate," Annals of the CIRP, 54/1:479-482, 2005.

4. Schmitt R., Niggemann C., "Uncertainty in measurement for X-ray-computed tomography using calibrated work pieces," Measurement Science and Technology, 21 (5), 2010.

5. Dewulf W, Tan Y, Kiekens K "Sense and non-sense of beam hardening correction in CT metrology," Annals of the CIRP, Manufacturing Technology, Vol.61 (1): 495-498, 2012.

6. Krumm M, Kasperl S, Franz M "Referenceless Beam Hardening Correction in 3D Computed Tomography Images of Multi-Material Objects," 17th World Conf. on Non-destructive Testing, Shanghai, China, 2008.

7. Amirkhanov A, Heinzl C, Reiter M, Kastner J, Groller E "Projection-Based Metal-Artifact Reduction for Industrial 3D X-ray Computed Tomography,” IEEE Trans. on Visualization and Computer Graphics, Vol 17, 2011.

8. Van.de.Casteele E. "Model-based Approach for Beam Hardening Correction and Resolution Measurements in Microtomography", PhD Thesis, University of Antwerp, 2004.

9. "Sources of Attenuation" www.net-ed.org/EducationResources/CommunityCollege/Radiography/Physics/attenuation.htm

10. Kyriakou Y, Kalender Willi A “X-ray scatter data for flat-panel detector CT," Physica Medica, Vol. 23 (1): 3-15, 2007

11. Welkenhuyzen F, Boeckmans B, Kruth JP, Dewulf W, Voet A "Simulation of X-ray projection images for dimensional CT metrology," 5th international conference on optical measurement techniques for structures and systems, p.477-487, Antwerp, 2012.

12. Bellon C, Deresch A, Gollwitzer C, Jaenisch G-R "Radiographic Simulator aRTist: Version 2," 18th World Conf. on Non-destructive Testing, Durban, South Africa, 2012. 